\title{
Improving the Accuracy of the Video Popularity Prediction Models through User Grouping and Video Popularity Classification
}

\author{
MASOUD HASSANPOUR and SEYED AMIR HOSEINITABATABAEI, Institute for Communica- \\ tion Systems (ICS), University of Surrey \\ PAYAM BARNAGHI, Centre for Vision, Speech and Signal Processing (CVSSP), University of Surrey \\ RAHIM TAFAZOLLI, Institute for Communication Systems (ICS), University of Surrey
}

This paper proposes a novel approach for enhancing the video popularity prediction models. Using the proposed approach, we enhance three popularity prediction techniques that outperform the accuracy of the prior state-of-the-art solutions. The major components of the proposed approach are two novel mechanisms for "user grouping" and "content classification". The user grouping method is an unsupervised clustering approach that divides the users into an adequate number of user groups with similar interests. The content classification approach identifies the classes of videos with similar popularity growth trends. To predict the popularity of the newly-released videos, our proposed popularity prediction model trains its parameters in each user group and its associated video popularity classes. Evaluations are performed through a 5-fold cross validation and on a dataset containing one month video request records of 26,706 number of BBC iPlayer users. Using the proposed grouping technique, user groups of similar interest and up to 2 video popularity classes for each user group were detected. Our analysis shows that the accuracy of the proposed solution outperforms the state-of-the-art including SH, ML, MRBF models on average by $45 \%$, 33\% and 24\%, respectively. Finally, we discuss how various systems in the network and service management domain such as cache deployment, advertising and video broadcasting technologies benefit from our findings to illustrate the implications.

CCS Concepts: • Theory of computation $\rightarrow$ Design and analysis of algorithms ; Information systems $\rightarrow$ Information systems applications ; • Networks $\rightarrow$ Network management;

Additional Key Words and Phrases: Popularity Prediction Models, Video Popularity Classification, User Grouping, Network and Service Management Applications.

\section{ACM Reference Format:}

Masoud Hassanpour, Seyed Amir Hoseinitabatabaei, Payam Barnaghi, and Rahim Tafazolli. 2019. Improving the Accuracy of the Video Popularity Prediction Models through User Grouping and Video Popularity Classification. ACM Trans. Web 1, 1, Article 39 (November 2019), 27 pages. https://doi.org/0000001.0000001

\section{INTRODUCTION}

The traditional reactive networking systems and management approaches are gradually replaced by smarter alternatives that take a preemptive rather than reactive approach to network management issues. Besides, demand for video content over the Internet is experiencing an exceptional growth.

Authors' addresses: Masoud Hassanpour; Seyed Amir Hoseinitabatabaei, Institute for Communication Systems (ICS), University of Surrey, Guildford, Surrey, GU2 7XH, England; Payam Barnaghi, Centre for Vision, Speech and Signal Processing (CVSSP), University of Surrey, Guildford, Surrey, GU2 7XH, England; Rahim Tafazolli, Institute for Communication Systems (ICS), University of Surrey, Guildford, Surrey, GU2 7XH, England.

Permission to make digital or hard copies of all or part of this work for personal or classroom use is granted without fee provided that copies are not made or distributed for profit or commercial advantage and that copies bear this notice and the full citation on the first page. Copyrights for components of this work owned by others than the author(s) must be honored. Abstracting with credit is permitted. To copy otherwise, or republish, to post on servers or to redistribute to lists, requires prior specific permission and/or a fee. Request permissions from permissions@acm.org.

(c) 2019 Copyright held by the owner/author(s). Publication rights licensed to ACM.

1559-1131/2019/11-ART39 \$15.00

https://doi.org/0000001.0000001

ACM Trans. Web, Vol. 1, No. 1, Article 39. Publication date: November 2019. 
While video pervades almost every application, it also puts tremendous pressure in the network to support users, that are expecting a high quality of experience, in a cost-effective manner.

The convergence of efforts in both smart network management and improving video content delivery, has sparked a new wave of research for Video Popularity Prediction Models (V-PPMs). V-PPMs are important tools for provisioning the demand for video contents and are widely used in networking paradigm. For example, predicting the popularity of videos has been proposed as an efficient method to improve the performance of content caching systems [25] and broadcasting technologies [39]. Direct benefits of this are to reduce latency and improve network traffic management [26]. Apart from the mentioned network-related applications, predicting which video contents will receive a large volume of requests is important for marketing agencies. An advertisement attracts more users if it is targeted to the most relevant audience [10].

The efficiency of the above-mentioned applications depends on the accuracy of the employed popularity prediction model [20]. As a result, finding new influential factors that have impact on the performance of a popularity prediction model and proposing a model that utilises the factors to improve the accuracy of the predictions of the existing V-PPMs is highly demanded.

In this paper, we propose a novel approach for enhancing V-PPMs ${ }^{1}$. Through our empirical evaluations, We show that the proposed approach significantly improves the performance of three well-known existing works. The approach takes two key factors that have a significant impact on the trend of the popularity into consideration. Herein, the trend of popularity is defined as how fast the number of users who are requesting a specific video changes over time.

Recent studies have proposed group-level popularity prediction as an approach which is more effective than user-level and population-level predictions [15]. Group-level predictions are less noisy than user-level predictions and in the same time, more accurate than population levels. On the other hand, in terms of video content consumption, users organise themselves into the groups with similar interests. This is the main reason for the success of Collaborative Filtering recommender systems [2]. Within the groups, users usually show quite consistent reactions to the newly-released videos [15].

In this study, we further hypothesise that there is a correlation between the level of the user interest in a specific video and the user reaction time. Hereby, we refer to the time between video release and the first request of the user as the user reaction time.

A direct implication of the above discussion is that grouping users based on the similarity of their previous video requests and customising the popularity prediction model parameters to the characteristics of each user group results in more accurate popularity predictions. According to this, the first factor of our proposed approach identifies user groups with similar interests, trains the prediction model parameters in each group and predicts the popularity of each video in each user group.

In addition, considering user interests enables us to predict the video popularity in a subset of population that are interested in a specific video. Almost all of the existing V-PPMs predict the popularity of a video in the whole population.

On the other hand, it has been shown that there are different distinct patterns in the popularity growth of videos [8]. Herein, we define the popularity growth of a video as how fast the number of requests received for a video grows. The distinct patterns can be used to classify videos into popularity classes. Therefore, the second factor of the proposed approach identifies the popularity classes and adapts the popularity prediction model parameters to these patterns can improve the accuracy of the popularity prediction models.

\footnotetext{
${ }^{1}$ Although the proposed method can be used to predict the popularity of all types of online contents, the method is more suitable for online videos. So, we limit our discussions in this paper to the online videos.
} 
Improving the Accuracy of the Video Popularity Prediction Models through User Grouping and Video Popularity Classification

Considering the above-discussed points, we propose a novel approach for enhancing V-PPMs which incorporates both the user groups and video popularity classes to model parameters. The proposed approach comprises the following processes:

- Extracting the underlying similarities between the video requests of users and utilising it to put the users into different groups.

- Extracting the underlying similarities between the pattern of the popularity growth of different videos and using it to assign the videos into classes with similar popularity growth patterns.

- Training the optimal values of the parameters of the popularity prediction model in each user group and its associated video popularity classes.

We utilise our approach to enhance three well-known state-of-the-art popularity prediction models, namely Szabo-Huberman (SH) [32], Multivariate Linear (ML) [29] and MRBF models [29]. It is worth to be emphasised that we utilise these models to present a proof-of-concept for applicability of the proposed approach; however, the proposed approach can be used to enhance any popularity prediction model. Figure 1 presents an overview of the proposed approach.

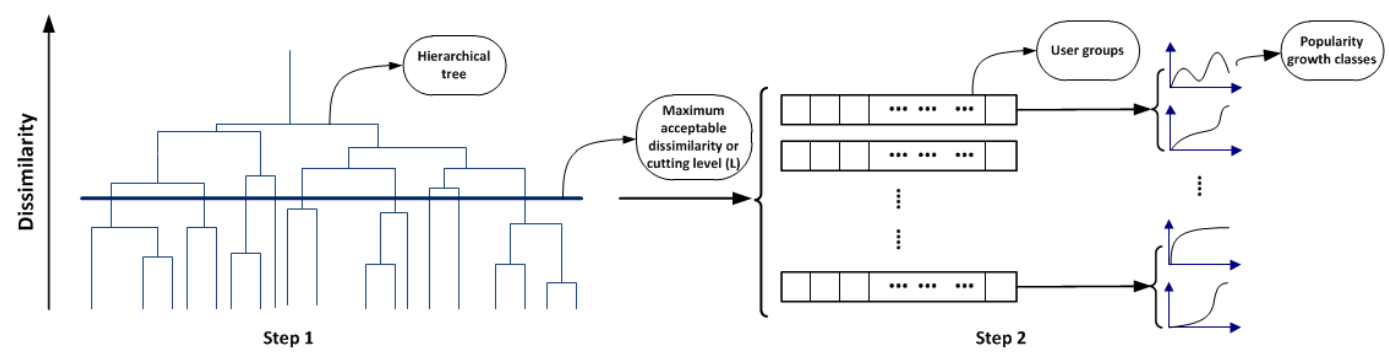

Fig. 1. In step 1, using the distance (dissimilarity) between users, a hierarchical tree is constructed. The horizontal links between every two different parts of the depicted tree show the dissimilarity (distance) between these parts. Using the entropy-based measure, the maximum acceptable dissimilarity is found and the tree is cut at this level; the latter process assigns the users into several groups. In step 2, within each group, the proposed technique finds different popularity growth trends and their associated model's parameters.

To study and evaluate the proposed approach, we utilise a dataset containing one-month video request records of the users of $\mathrm{BBC}$ iPlayer. we compare the prediction errors resulted from the proposed model with the errors of the conventional counterparts. Our results show that the proposed approach is able to significantly improve the employed popularity prediction models.

Our contributions in this paper can be summarised as follows,

- We propose a novel approach to enhance the existing V-PPMs. The approach contains two steps:

(1) We propose to group users based on their interest as an effective factor for predicting the group popularity of online videos. In doing so, we propose an unsupervised technique to determine the optimal number of user groups. The optimal number of user groups is determined such that the predictability of the user behaviours in the constructed groups is maximised.

(2) We propose to classify videos into the classes with similar popularity growth trends. For this purpose, we develop a novel video popularity classification technique that minimises the mean and range of the prediction errors. 
- We elaborate on our approach and show how the user grouping and video classification are used to enhance a popularity prediction model by considering its parameters as a function of the user group and popularity class number.

- We provide a closed-form expression to find the optimum parameters of the proposed popularity prediction models.

- We present an extensive evaluation to test the impact of the proposed approach on the performance of the state-of-the-art popularity prediction models.

The properties of our proposed approach and the presented findings in this study have a number of implications for improving the network and service management applications of a popularity prediction algorithm. The most familiar examples of the applications are broadcasting, content caching, Device to Device caching and marketing industries. In brief, the applications ought to consider the characteristics and spatial distribution of user groups, population of users in each group, and video popularity classes to improve the performance of the mentioned applications. The implications are discussed in more detail in section 6.

The rest of this paper is organised as follows. Section 2 discusses the related works. Section 3 discusses why we should expect that considering user groups and popularity classes improves the V-PPMs . The methodology for the proposed approach is presented in section 4. Section 4.1 discusses our unsupervised grouping method. The proposed video classification technique is explained in section 4.2. In section 4.3, we present a detailed discussion on how our approach can be used to enhance three V-PPMs. Discussion on the used dataset and the evaluation results are presented in section 5. Section 6 discusses the implications resulted from our proposed approach to improve the potential applications of the V-PPMs. Finally, we conclude this paper in section 7 and discuss the open issues.

\section{RELATED WORKS}

\subsection{Popularity prediction techniques}

There is a wide range of studies that aim to model the popularity of the online videos. These studies define the popularity of online videos is as the number of views that the videos receive over time [34].

The online videos can be classified into two categories of User Generated Contents (UGCs) [34] and on-demand videos. The UGCs are generated by users (e.g. YouTube videos); while, on-demand videos are released on a regular-basis by content providers (e.g. TV series).

In the literature, two main approaches are used for video popularity modeling including finding the best fit to the view count distribution and correlation-based methods. These approaches are explained in the following sections.

\section{Finding the best fit to the view count distribution}

In this approach, researchers try to find the best distribution function that provides a good estimation of the view count distribution (or the number of views) over time. For instance, in different studies, log-normal [3], Zipf-like [7], power-law with exponential cut-off [4], Gamma [5], and Weibull [6] distributions have been proposed as the best-fitted distributions to the view count of online videos. Observing different distributions can be due to the datasets that these studies used. Considering this point, Avramova et al. [1] studied the evolution of popularity of online videos in three different datasets and showed that the popularity evolution can be described as power-law or exponential distributions. Wu et al. [35] considered two different driving forces in the evolution of the view count distribution. They divided users based on those who are interested in a newly-released video 
and those who receive the recommendation from the first group. Considering different parameters for each group, $\mathrm{Wu}$ et al. modeled the trend of received popularity for a newly-released video.

The major shortcoming of the discussed studies is that the above-mentioned distributions only capture the general trend of the popularity growth of online videos. However, there may be differences in the trends of the view count distributions of videos. In [9] and [8], the authors found four distinct patterns for describing the popularity growth of videos which were classified as: memoryless, junks, viral and quality. Figueiredo [11] utilised these four classes and used a $\mathrm{K}$-spectral clustering to classify the view count distributions of videos over time to predict the popularity of YouTube videos. In another study, Li et al. [19] employed a random forest classifier to correlate a number of video features (more than 50 features) with the class of videos.

\section{Correlation-based methods}

In a quite different approach, some studies have focussed on capturing and exploiting the correlation between the early popularity and the received popularity in the future. Szabo and Huberman [32] used the mentioned correlation and proposed a linear regression model that relates the number of received requests until a given time in the future to the number of requests received up to a given time before that. Pinto et al. [29] extended the model proposed in [32] to a Multivariate Linear model (ML model); the ML model correlates the number of requests received in a given time to the number of requests received till several point of times in the past. Considering the differences in the trend of popularity growth, the authors also proposed MRBF model that extends ML model by a new term that considers the similarities of the trend of the popularity growth of different videos. LARM (a Lifetime Aware Regression Model) [24] is another regression based model which utilises the life time of videos to improve the accuracy of its predictions. To estimate the life time of videos, a prediction method is proposed in [24]. The evaluation results show that LARM outperforms MRBF by around $20 \%$.

Considering the correlations between received popularity at different times, $\mathrm{Wu}$ et al. [36] developed a model based on Reservoir Computing (RC), which is a neural network technique. The authors observed that there is a high correlation between some definite time intervals in the past and future. Tan et al. [33] proposed a model based on Pure Birth Process, which is a time-dependent Markov Chain model, to predict the accumulated future view count of online videos.

The above-explained models consider the same set of parameters for all videos. As a result, these models still do not capture all the characteristics of the popularity trend of different videos, which in return degrade the prediction accuracy. To address this shortcoming, some studies have attempted to find some other features that have impact on the popularity growth of videos. For example, it has been shown that there is a noticeable correlation between the social influence of uploader (defined as the number of followers) [28], [27], the popular topic extracted from social streams [30], visual sentiments features [37], [12] of videos and the popularity of the videos. The above-mentioned features can be utilised to find the best model (or distribution) that describes the popularity growth of different videos [28], [38] or the sudden rises in the popularity of a video [30].

Finally, Hoang et al. [15] have proposed a method for predicting group-level popularity of online contents, e.g. videos, news or posts, in social networks. Although their method takes the interests of users into consideration, their approach for user grouping is different from ours and they do not consider the popularity class of videos. Additionally, their proposed method is suitable for social networks, while our proposed approach is applicable in more generic solutions. To be more precise, in [15], k-way graph partitioning technique is employed to group users. This technique is applicable when we have constructed a popularity graph (as described in [15]) and is different from our grouping technique. We can classify the advantages of our proposed grouping technique in comparison with the one in [15] in 3 following points. 
- The technique used in [15] has to have number of clusters in advance; while, our technique finds the number of clusters such that the predictability of user groups has its maximum value.

- On the other hand, the grouping technique used in [15] has a constraint that all user groups should approximately have similar sizes. We argue that this may result in some inaccuracy as the size of the user groups may be different.

- The utilised grouping technique in [15] is limited to the occasions where there is a time series of behaviors and is suitable for social networks, while our proposed approach is applicable in more generic cases.

Our proposed approach in this paper can be utilised to enhance all the above-explained V-PMMs. Our approach takes into account two factors that have not considered in the previous V-PPMs.

First, the proposed algorithm is the first V-PPM that takes the interest of users into consideration and predicts the popularity of each video in user groups instead of the whole user population. As for finding user groups, we introduce a new entropy-based method for estimating the optimal number of user groups. Justifications on the novelty of the proposed method is provided in section 2.2 .

Second, inspired by the findings of [8], the proposed algorithm considers the existence of the different popularity classes of videos and leverage this information to improve the accuracy. To do this, the algorithm benefits from a novel video popularity classification technique to classify the videos into a number of classes. Our algorithm only utilises the growth of the popularity of a video in the early times after release time to identify the popularity class of the video.

\subsection{Entropy-based grouping techniques}

There are several clustering algorithms in the literature that have used entropy; however, the majority of the algorithms still need an input parameter to determine the number of clusters. One of the most well-known entropy-based clustering approaches is to add a entropy based fuzzy membership to the clustering algorithm, e.g. [18] and [21]. This approach adds a new entropy-based term to the objective function of k-means clustering. The new entropy-based terms depend on the probability that a data point belongs to each cluster. Liao et al. [23] developed a similar fuzzy membership clustering method with a weight parameter on the membership entropy term for categorical data. Their method uses an input threshold maximum dissimilarity to find the best number of clusters. In [22], Liang et al. used within and between cluster entropy and proposed entropy-based measures to find the worst clusters in a dataset containing both categorical and numerical attributes. The authors also proposed a cluster validity index, which does not depend on entropy, to find the best number of clusters in a dataset. However, except [22], the mentioned clustering method needs the number of clusters as input. Shu et al. [31] proposed a measure based on marginal differential entropy for $\mathrm{n}$-dimensional datasets. The measure considers the probability distribution function of the data points in each cluster alongside different dimensions and calculates the total marginal differential entropy. The best number of clusters is where the derivative of the total marginal differential entropy is less than a pre-determined threshold. The measure tries to find the clusters that have denser distributions and is suitable for density-based clustering techniques.

In this paper, we propose a novel method to find the optimum number of clusters. The method benefits from an entropy-based measure. The proposed method can be used to enhance most of the existing clustering techniques and provides two main novel advantages,

- The proposed entropy-based method determines the best number of groups in a way that maximises the predictability of the group behaviours. This point makes our proposed method suitable to be used in a predictive algorithm. 
- The proposed method can be directly used to enhance almost all of the existing clustering technique, including the above-explained techniques. The method enables a clustering technique to determine the best number of clusters. Different from the clustering techniques presented in [18], [21] and [23], the proposed method does not make any change in the objective function of the clustering algorithm.

\section{MODEL JUSTIFICATION}

This section is dedicated to provide a discussion on why considering the user groups with similar interests and popularity classes of videos enables us to make a better estimation of the future popularity of a newly-released video. Before beginning our discussion, we need to first define the definition that we considered for popularity of a video. There are two ways for defining popularity of a video. The ways are,

- View count received for each video,

- Number of unique users who have requested each video.

Each definition has its own advantages and disadvantages. In our paper, we considered the number of the unique users requesting a video as the popularity of that video. Selecting this definition is because of 1) the applications of this kind of definition (e.g. advertisement purposes and improving Quality of Experience of users) and 2) the robustness of this number against changes in network condition. In addition, when the bad network condition does not have a significant impact, we do not lose the generality of our results by considering this definition. The reason is that in this case the number of times that a user request a particular video only depends on her interest; and for the videos with the same attractiveness for the user, this behavior may occur too.

To foresee the number of requests received for a specific video in a future time, different studies have proposed different models to estimate the distribution of the received requests. We call the distribution of the received requests as the request count distribution and denote the normalised aggregated request count distribution for video $v$ by $P_{T}^{v}\left(t_{v}\right)$; where, $t_{v}$ is the time passed since the video $v$ has been released. $P_{T}^{v}\left(t_{v}\right)$ is normalised by number of all users.

The majority of the V-PPMs try to directly estimate $P_{T}^{v}\left(t_{v}\right)$ for the whole population of users. However, this may result in some inaccuracies specially when users have different reaction time distributions to different videos. So, we argue that estimating the request count distribution in the groups of users that have similar reaction time distributions and doing weighted sum over the estimated request count distributions can provide a more accurate estimation for $P_{T}^{v}\left(t_{v}\right)$.

To find the users with similar reaction time distributions, we take into account three particularities of users' content requests.

- Recent studies on Collaborative Filtering recommender systems [2] suggest that users organise themselves in the groups with similar interests.

- Users usually show consistent reactions to a newly-released video within each user group [15]

- Users' reaction times in each group are different from the reactions of users in the other groups [14].

Inspired by the above discussion, we hypothesise that distinct groups of users that have different reaction time distributions can be identified by looking at their interest in different videos. As a result, we put the users that have requested similar videos into the same groups and claim that the formed user groups have quite similar reaction time distributions.

On the other hand, the studies in [9][8] have shown that the popularity growth patterns of videos can be classified into a number of popularity classes with distinct growth patterns. Therefore, 
we further propose considering various video classes in training popularity prediction model parameters improves the accuracy of the popularity predictions.

Finally, we propose that estimating the request count distribution in each user group for each video that has a specific popularity class and doing a weighted sum over all the obtained distributions is a more accurate method than estimating the request count distribution for the whole population of users. To support this claim, based on the above discussed points, one can conclude that the request count distribution changes for different user groups and video popularity classes. As the result, if we estimate the request count distribution of a video in each user group while we consider the dependency of this distribution to the popularity class of the video and sum over all the obtained distributions, we would achieve a more accurate estimation for the total distribution.

According to this, the following expression can be written for estimating the total request count distribution (denoted by $\widehat{P}_{T}^{v}\left(t_{v}\right)$ ).

$$
\widehat{P}_{T}^{v}\left(t_{v}\right)=\sum_{k=1}^{\left|G_{k}\right|} \omega_{k} \widehat{P}_{T}^{k, h}\left(t_{v}\right),
$$

where, $k$ denotes the $k^{t h}$ user group and $h$ denotes the $h^{t h}$ video popularity class number. In other words, $P C_{h}^{k}$ is the $h^{t h}$ popularity class of the videos in $k^{t h}$ user group; and $\widehat{P}_{T}^{k, h}\left(t_{v}\right)$ is the normalised request count distribution obtained in user group $k$ and is obtained when the popularity class $(h)$ of the video is considered; $\widehat{P}_{T}^{k, h}\left(t_{v}\right)$ is normalised by the number of users in group $k ; \omega_{k}$ is a weight parameter and depends on the proportion of users in the $k^{t h}$ group. Due to the way that we have normalised the distributions in 1, the weighted sum works and gives the number of requests received for a video divided by total number of users.

\section{METHODOLOGY AND THE PROPOSED TECHNIQUE}

Our proposed popularity prediction technique includes two parts called training and popularity prediction. In what follows, we discuss these parts in detail.

In the training part, we first find those users who have similar reaction time distributions to the newly-released videos. The hypothesis discussed in section 3 implies that users with similar interest may have similar reaction time distributions to the newly-released videos. As the result, we put users who have requested similar videos into the same groups.

Next, within the constructed user groups, our proposed solution finds the popularity classes that have similar popularity growth trends. The maximum and minimum of the early received popularities of each video popularity class are recorded as the characteristic features for identifying the video classes. We define the early received popularity as the number of requests that are received in each user group after the release of each video until a predefined time parameter (an input parameter that determines how much time later we would like to make the first prediction about the popularity of videos).

In the next step, the parameters of the popularity prediction model are trained in each video popularity class and user group. As a result, the parameters of the models are a function of user group and video class numbers.

The above-described steps until this stage can be used to enhance any popularity prediction model. To illustrate how we can employ the above steps to enhance a popularity prediction model, we have chosen three state-of-the-art models that are SH, ML and MRBF.

In the prediction part, first, the class of the newly-released videos in each user group is identified based on the popularity class characteristics (which were explained above). Next, the model parameters related to each user group and popularity class are used to predict the future popularity of the videos. 
To evaluate of our proposed popularity prediction algorithm, we compare the accuracy of our popularity prediction model with its conventional counterpart.

In the rest of this section, we discuss three main parts of the training phase of our proposed approach, i.e. user grouping, video classification and training model parameters as a function of the user groups and popularity classes.

\subsection{Grouping users based on their interests}

We group users based on the similarity between previously demanded videos. In the present section, we elaborate on the grouping (clustering) technique that we utilise to group users.

In brief, the proposed grouping technique employs the Manhattan distance [16] to find the distance between user interests and constructs a hierarchical tree. Then, the technique employs a novel entropy-based measure, to find the best number of clusters. In the following, we discuss our grouping method as well as the proposed entropy-based measure.

Suppose there is a set of users, denoted by $\{\mathbf{U}\}$, and a dataset containing the requests of the users for a set of $m$ videos, denoted by $\{\mathbf{V}\}$. We define a set of Request Vectors $\{\boldsymbol{R} \boldsymbol{V}\}$ containing $m$-dimensional binary Vectors (denoted by $\overrightarrow{R V}^{i}$ s). A request vector $\overrightarrow{R V}^{i}$ is assigned to the user $u_{i} \in\{\mathbf{U}\}$. Each element of $\overrightarrow{R V}^{i}$ determines whether user $u_{i}$ has requested a particular video or not. In this regard, $\overrightarrow{R V}{ }^{i}$ is a representation of each user's interest. A typical $\overrightarrow{R V}^{i}$ is as follows:

$$
\left.\overrightarrow{R V^{i}=} \begin{array}{c|c}
\text { Video \#1 } & 1 \\
\text { Video } \# 2 & 0 \\
\vdots & \vdots \\
\text { Video \#l } & 1 \\
\vdots & \vdots \\
\text { Video \#m } & 1
\end{array}\right],
$$

Using $\overrightarrow{R V}{ }^{i}$ and $\overrightarrow{R V}^{j}$, the distance between the interests of users $u_{i}$ and $u_{j}$ can be calculated. There are many methods for calculating the distance. Euclidean, Manhattan and Cosine distances are three well-known examples of these methods. We select Manhattan distance in this paper to measure the distance between the interests of two users. The Manhattan distance between two $\overrightarrow{R V}^{i} \mathrm{~s}$ is defined as follows,

$$
D_{i j}=\sum_{k}\left|R V_{k}^{i}-R V_{k}^{j}\right|
$$

in which, $R V_{k}^{i}$ is the $k^{t h}$ component of $\overrightarrow{R V}^{i}$. Hierarchical clustering [17] algorithm is then utilised to cluster the users.

Hierarchical clustering technique constructs a hierarchical tree based on the distances between users. The technique requires a cutting level as input to put users in different clusters.

We propose a method to use our entropy-based measure to find the optimum value of the cutting level. Since the entropy is a measure of the predictability of an event in a system (herein, requesting a video by users in a particular group), minimising total entropy leads to determining the number of groups (clusters) in which the events are more predictable. As a result, the method is suitable to be used in a predictive model. Below, we explain our proposed method in detail.

First, our proposed method cuts the hierarchical tree in a level, denoted by $L$ (step 1 in Fig. 1). So, it results in some user groups $G_{k}$. Next, the proposed method utilises Shannon entropy to calculate the entropy of the video requests of the users in $G_{k}$. Since in different cutting levels $L$ there are 
different user groups, the calculated entropy will be a function of $L$. The entropy for each user group can be calculated by,

$$
H^{k}(L)=-\sum_{v \in\{\mathbf{V}\}} P_{v}^{k}(L) \log _{2}\left(P_{v}^{k}(L)\right)
$$

where, $P_{v}^{k}(L)$ is the probability that video $v$ is requested in the user group $G_{k}$ and can be written as,

$$
P_{v}^{k}(L)=\frac{\text { Num of users who Requested video } v \text { in } G_{k}}{\text { Total Num of users in } G_{k}} .
$$

Calculating $P_{v}^{k}(L)$ in the user groups with only a small number of users can cause some inaccuracies. Due to this, we select a threshold as the minimum acceptable number of users in each group. This is to avoid small groups creation. A random behaviour is considered for those users who are in the groups that contain a number of users less than the threshold. Random behaviour in this context is equivalent to requesting the videos with the probability of $\frac{1}{2}$ (i.e. $P_{v}^{k}(L)=\frac{1}{2}$ ). The random behaviour results in maximum entropy and penalises the creation of the groups that fall below the minimum threshold size. The total entropy of the resulted groups is formulated as,

$$
H_{T}(L)=\sum_{k=1}^{K} \omega_{k} H^{k}(L) .
$$

where, $\mathrm{K}$ is the number of groups resulted in each cutting level $(L)$ and $\omega_{k}$ is the proportion of all users in group $k$. The cutting level $L$ (or equivalently, number of groups $K$ ) in which a global minimum occurs is selected as the best number of groups. Searching for the optimal value of the cutting level needs an upper bound on $L$ values which is denoted as $L_{\text {max }}$. A reasonable guess for $L_{\max }$ can be the maximum distance between users (maximum of $D_{i j}$ ). The pseudo-code of the proposed grouping technique is presented in the Algorithm 1.

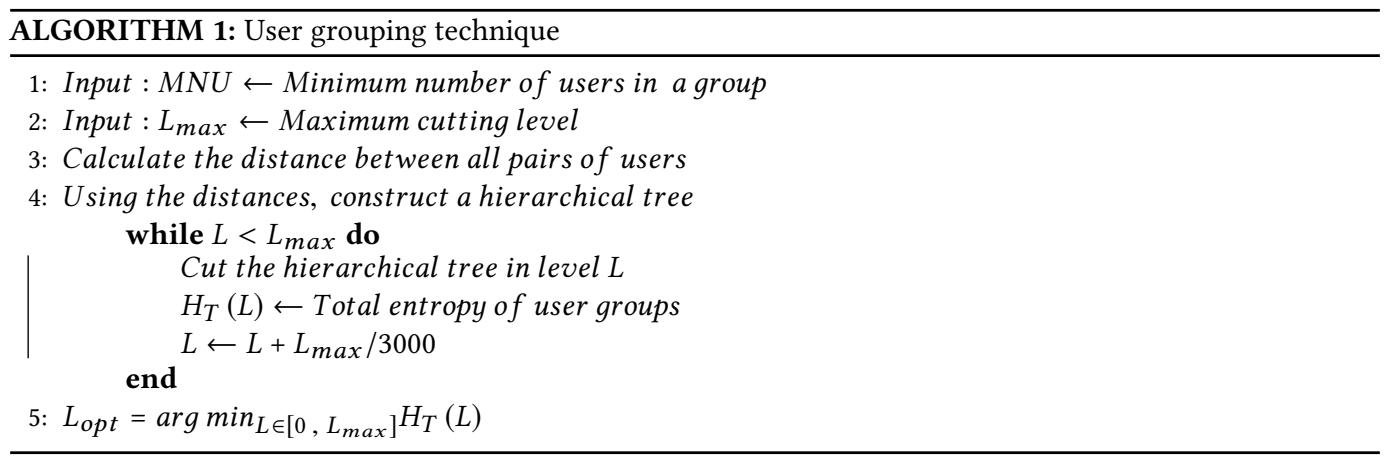

\subsection{The proposed video classification technique}

This section discusses our proposed video popularity classification technique. We utilise this method to divide the videos into distinct popularity classes with similar popularity growth trend.

The proposed classification method needs a popularity prediction model in its core. The method divides videos into a number of popularity classes and trains a distinct set of the parameters of the selected popularity prediction model in each popularity class. The method then utilises the training dataset and the trained parameters to find the Relative Square Error (RSE) of the popularity model. Next, the method finds the popularity classes such that the sum of total RSEs for all videos and total Standard Error $(S E)$ of the RSEs are minimised. The best choice for the popularity prediction model 
Improving the Accuracy of the Video Popularity Prediction Models through User Grouping and Video Popularity Classification

selected by the classification technique is to use the same model that we wish to enhance. For example, when we want to enhance SH model, the classification method utilises the same model (SH model). Nevertheless, one may use one of the regression models (e.g. SH or ML) to classify the videos and consider it as the sub-optimal classification to be used in other models.

In brief, the classification method starts with setting a number of thresholds on request counts that we call popularity levels. By comparing the early popularity (as was defined in section 4) of each video with these popularity levels, the method puts the videos into a number of popularity classes. Next, the method calculates a Cost Function that depends on the above-discussed RSEs for the current configuration of the popularity levels. The method changes the popularity levels and calculates the Cost Function for each configuration. The Cost Function takes the errors as well as the standard error of the predictions into consideration. The classification method repeats this process with different configurations until the configuration of the popularity levels that minimises the Cost Function is found. Finally, the optimum values of the popularity levels are considered as the levels for classifying the videos. In what follows, the ordered steps of the proposed classification method is explained in more details.

The video classification process utilises the early popularity, denoted by $E P_{j}^{k}$ as input. As mentioned in section 4, the early popularity is defined as the number of requests that are received in $k^{t h}$ user group after the release of the $j^{t h}$ video until a predefined time parameter. Then, the classification technique gets the maximum acceptable number of classes for the $k^{\text {th }}$ user group, denoted by $N_{c}^{k}$, as input.

In each repetition, which is labeled by $i$, the classification method randomly generates $N_{c}^{k}-1$ popularity levels, denoted by $P_{l}^{k}$, for each user group. The popularity levels are generated such that they belong to the interval $\left[0, P_{\max }^{k}\right) . P_{\max }^{k}$ is the maximum early popularity of all videos in group $G_{k}$. The classification process is repeated $I_{\max }$ times to find the video popularity classes through a stochastic search. To give a criterion for selecting a value for $I_{\max }, I_{\max }$ should be at least equal to $\left(P_{\max }^{k} / N_{c}^{k}\right) \times$ Num of videos.

Since we need enough number of videos to find the optimal values of model parameters, our proposed classification technique imposes a minimum for the number of videos in each class. By this way, the technique prevents the classes with small number of videos. If there is a popularity class with the number of videos less than this minimum number, the classification technique combines this class to the previous popularity class; unless it happens for the first class. In this case, the class is merged to the next class. This reduces the total number of classes by 1 (i.e. $N_{c}^{k}$ is updated as $\left.N_{c}^{k} \equiv N_{c}^{k}-1\right)$. This procedure continues till the number of videos in all classes are greater than the minimum number.

Next, the early popularity levels are sorted in an increasing order, i.e. $P_{0}^{k}=0<P_{1}^{k}<P_{2}^{k}<$ $\cdots<P_{N_{c}^{k}-1}^{k}<P_{\text {max }}^{k}$. The video classification method assigns the videos to $N_{c}^{k}$ popularity classes, denoted by $P C_{i}^{k}$. If the early popularity received for a video $v_{j}$ in group $G_{k}$ is in the interval $\left[P_{h-1}^{k}, P_{h}^{k}\right)$, the video is assigned to the popularity class $P C_{h}^{k}$. So, the popularity classes are defined as

$$
P C_{h}^{k}=\left\{\forall v_{j} \mid P_{h-1}^{k}<E P_{j}^{k} \leq P_{h}^{k}\right\},
$$

According to Eq. 7, the popularity class $P C_{h}^{k}$ is a function of user group $G_{k}$; and differs from one group to another one.

Next, the classification technique finds the optimal values of the parameters of the selected regression model in each user group and each popularity class such that the total RSE is minimised. The total RSE actually determines how well the regression model has fitted to the request count 
distribution of all videos in training dataset. Then, the classification method puts the discussed $R S E$ s for the videos in each popularity class $\left(N_{c}^{k}\right)$ and a specific user group $\left(G_{k}\right)$ into a set denoted by $R S E^{k, h}$.

In the next step, the proposed technique averages over the $R S E^{k, h} \mathrm{~s}$ in each popularity class. We denote the mean of $R S E^{k, h}$ s by $M R S E^{k, h}$. NTRSE $E^{k}$ is the normalised total RSE and is defined as,

$$
\operatorname{NTRSE}_{i}^{k}=\sum_{h=1}^{N_{c}^{k}} \frac{M R S E^{k, h}}{\max \left(M R S E^{k}\right)-\min \left(M R S E^{k}\right)},
$$

where, $M R S E^{k}$ is defined as,

$$
\operatorname{MRSE}^{k}=\left(M R S E^{k, 1}, \operatorname{MRSE}^{k, 2}, \cdots, \operatorname{MRSE}_{k, N_{c}^{k}}\right),
$$

$N T R S E_{i}^{k}$ provides the mean of the RSEs associated to a particular classification given by Eq. 7 .

According to the central limit theorem, the limited sample size (i.e. limited number of RSEs in the $R S E^{k, h}$ set) causes an error in the estimation of the $M R S E^{k, h}$. This error is called Standard Error and is denoted by $S E$. We denote the $S E$ of $M R S E^{k, h}$ as $S E^{k, h}$ that is calculated as,

$$
S E^{k, h}=\frac{1}{\sqrt{\left|R S E^{k, h}\right|-1}} \operatorname{Std}\left(R S E^{k, h}\right)
$$

in which, $S t d\left(R S E^{k, h}\right)$ and $\left|R S E^{k, h}\right|$ are the Standard Deviation and size of $R S E^{k, h}$, respectively. We define the normalised total Standard Error of all RSEs as,

$$
N T S E_{i}=\sum_{h=1}^{N_{c}^{k}} \frac{S E^{k, h}}{\max \left(S E^{k}\right)-\min \left(S E^{k}\right)},
$$

where $S E^{k}=\left(S E^{k, 1}, S E^{k, 2}, \cdots, S E^{k, N_{c}^{k}}\right)$. The reason why we normalise $N T R S E_{i}^{k}$ and $N T S E_{i}^{k}$ is that all of their values have the same order of magnitudes. Since both of the above-explained errors should be considered, hence, we define Cost Function (CF) below,

$$
C F_{i}=\frac{1}{N_{c}^{k}}\left(N T R S E_{i}+N T S E_{i}\right) .
$$

In order to obtain the best classification of videos, we need to find the number $i$ in which the Cost Function presented in Eq. 12 has the minimum value. The proposed technique finds the minimum value of the Cost Function and the associated popularity levels for each group (i.e. $P_{i}^{k}$ ). The found popularity levels are used in the prediction phase to assign the newly-released videos to a suitable popularity class. The pseudo-code for the classification technique is presented in the algorithm 2 .

\subsection{Training model parameters as a function of user groups and popularity classes}

As discussed previously, we can use the user grouping and video popularity classification techniques, presented in the sections 4.1 and 4.2, to enhance any popularity prediction method. To elaborate on how the proposed approach can be employed to enhance a popularity prediction model, we have chosen three well-known popularity prediction models, namely $\mathrm{SH}$, ML and MRBF models.

This section presents a discussion on how we utilise these models along with the user groups and popularity classes to improve these popularity prediction models. In what follows, we clarify how we train the parameters of the proposed popularity prediction models as a function of the user group and popularity class numbers. For each model, we also present a closed-form expression for the optimal values of their parameters. 


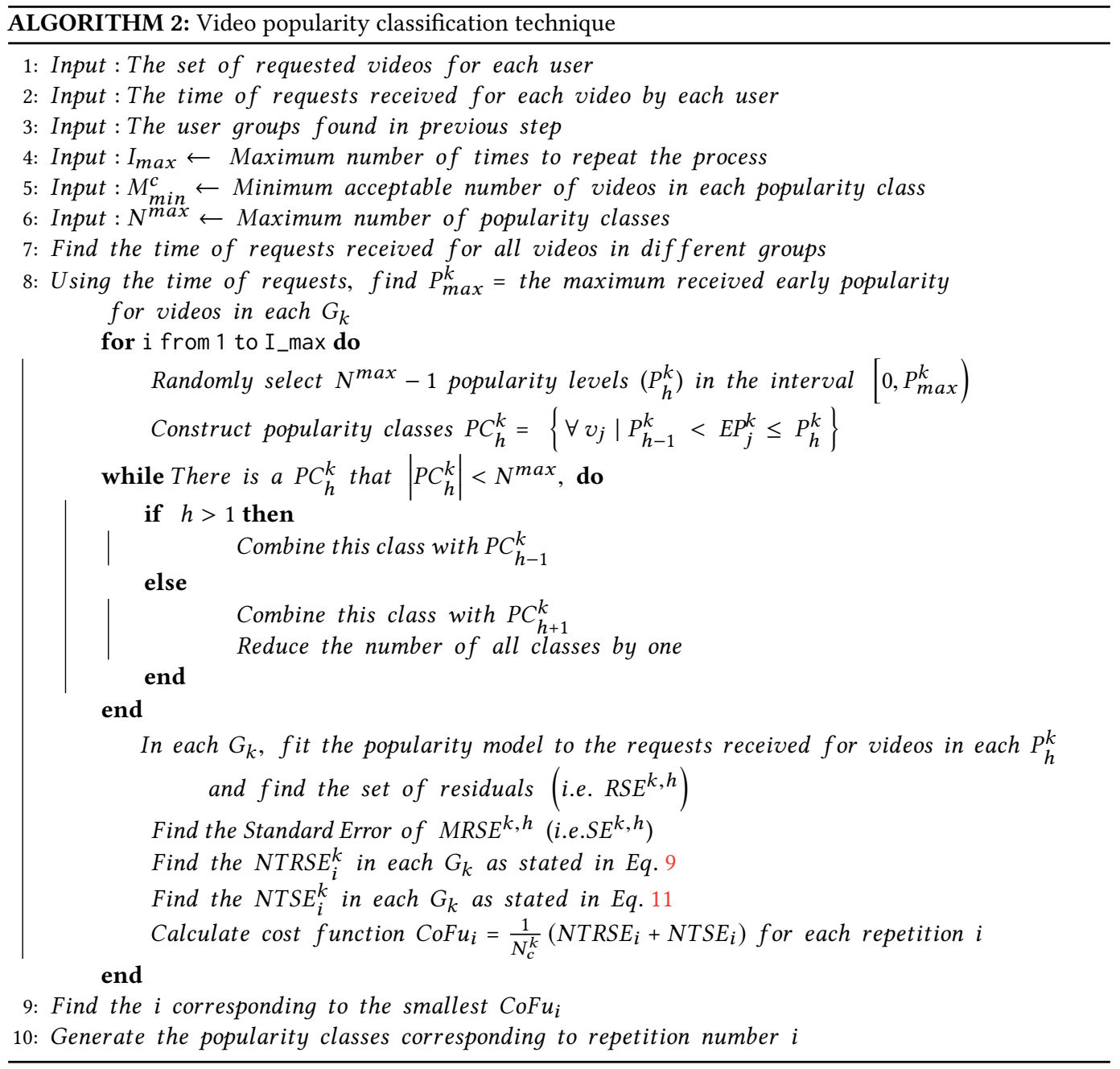

\section{Enhancing Szabo-Huberman model}

Szabo and Huberman [32] proved that there is a significant linear dependency between the logtransformed of the popularities of online videos in the present and future times. According to the mentioned point, we linearly correlate the number of requests received for a specific video $(v)$ in $t_{j}$ to the number of requests that this video has received up to a specific time in the past (i.e. $\left.t_{i}\right)$ in each user group $\left(G_{k}\right)$ and popularity class $\left(P C_{h}^{k}\right)$. So, we have,

$$
\widehat{N}_{t_{j}}^{v, k}=\alpha_{k, h}\left(t_{j}, t_{i}\right) N_{t_{i}}^{v, k},
$$

in which $v$ belongs to popularity class $P C_{h}^{k} ; \widehat{N}_{t_{j}}^{v, k}$ is the predicted number of requests in time $t_{j}$ for video $v$ (that is the request count distribution discussed in section 3) in group $G_{k} ; N_{t_{i}}^{v, k}$ is the actual number of requests received up to the time $t_{i}$ for video $v$ in group $G_{k}$; and $\alpha_{k, h}\left(t_{j}, t_{i}\right) \mathrm{s}$ are the model parameters that should be trained. It is evident that $\alpha_{k, h}\left(t_{j}, t_{i}\right) \mathrm{s}$ are a function of user group and popularity class number and need to be trained in each user group and popularity class. 


\section{Optimal values of $\alpha_{k, h}$ parameters}

Minimising Relative Squared Error with respect to $\alpha_{k, h}$ can be utilised to train the $\alpha_{k, h}$ parameter[32]. Considering this, the RSE of the predictions made by Eq. 13 is defined as,

$$
R S E=\frac{1}{\left|v \in P C_{h}^{k}\right|} \sum_{v \in P C_{h}^{k}}\left(\frac{\widehat{N}_{t_{j}}^{v, k}}{N_{t_{j}}^{v, k}}-1\right)^{2} .
$$

where, $N_{t_{j}}^{v, k}$ is the actual number of unique users that have requested video $v$ till time $t_{j}$ in group $G_{k}$. So, in calculating $N_{t_{j}}^{v, k}$, if a specific user requests video $v$ several times, we count all of those requests once for that user. $\left|v \in P C_{h}^{k}\right|$ is the number of videos in the popularity class $P C_{h}^{k}$. Substituting Eq. 13 into Eq. 14 will lead us to,

$$
R S E=\frac{1}{\left|v \in P C_{h}^{k}\right|} \sum_{v \in P C_{h}^{k}}\left(\frac{\alpha_{k, h}\left(t_{j}, t_{i}\right) N_{t_{i}}^{v, k}}{N_{t_{j}}^{v, k}}-1\right)^{2} .
$$

The RSE given by Eq. 15 is calculated in the user group $k$ and its associated video popularity class $h$. Similar to Szabo and Huberman [32], we provide the optimal value of $\alpha_{k, h}$ that minimises RSE by taking the derivative of the RSE with respect to $\alpha_{k, h}$ and setting it to zero. The optimal value of $\alpha_{k, h}$ is given by,

$$
\alpha_{k, h}\left(t_{j}, t_{i}\right)=\frac{\sum_{v \in P C_{h}^{k}} \frac{N_{t_{i}}^{v, k}}{N_{t_{j}}^{v, k}}}{\sum_{v \in P C_{h}^{k}}\left(\frac{N_{t_{i}}^{v, k}}{N_{t_{j}}^{v, k}}\right)^{2}} .
$$

Eq. 16 can be utilised to find the optimal value of $\alpha_{k, h}$ for the popularity class $P C_{h}^{k}$ and user group $G_{k}$. Since the $R S E$ is a second order equation the expression Eq. 16 provides the optimal value of $\alpha_{k, h}$ that globally minimises $R S E$.

\section{Enhancing Multivariate Linear (ML) model}

Pinto et al. [29] extended the $\mathrm{SH}$ model such that the number of requests received for each video until a time $t_{j}$ is linearly correlated to the actual number of requests received for that video in the all time intervals of $\left[t_{i-1}, t_{i}\right]$ before $t_{j}$. Considering the Multivariate linear regression model and the proposed approach in this paper, the model below can be written for the number of requests received for each video in each user group,

$$
\widehat{N}_{t_{j}}^{v, k}=\sum_{i=1}^{j-1} \theta_{j, i}^{k, h} X_{t_{i}}^{v, k}
$$

where $X_{t_{i}}^{v, k}$ is the number of requests received for the video $v$ in group $k$ and $\theta_{j, i}^{k, h} \mathrm{~s}$ are the model parameters that are a function of popularity class $P C_{h}^{k}$ and user group $G_{k}$.

\section{Optimal values of $\theta_{j, i}^{k, h}$ parameters}

Similar to the procedure we followed in the previous section, minimising RSE with respect to $\theta_{j, i}^{k, h}$ may provide a method for learning $\theta_{j, i}^{k, h}$ parameters. It is worth to note that RSE should be 
Improving the Accuracy of the Video Popularity Prediction Models through User Grouping and Video Popularity Classification

calculated in each video popularity class and user groups. So, we can write the RSE as

$$
R S E=\frac{1}{\left|v \in P C_{h}^{k}\right|} \sum_{v \in P C_{h}^{k}}\left(\frac{\sum_{i=1}^{j-1} \theta_{j, i}^{k, h} X_{t_{i}}^{v, k}}{N_{t_{j}}^{v, k}}-1\right)^{2},
$$

To obtain the optimum value of $R S E$, we need to set its derivative with respect to $\theta_{j, i}^{k, h}$ to zero. If we do this and define the following matrices,

$$
\begin{aligned}
\vec{\theta}^{k, h} & \equiv\left(\theta_{j, 1}^{k, h}, \theta_{j, 2}^{k, h}, \cdots, \theta_{j, j-1}^{k, h}\right), \\
B_{i}^{k, h} & \equiv \sum_{v \in P C_{h}^{k}}\left(\frac{X_{t_{i}}^{v, k}}{N_{t_{j}}^{v, k}}\right), \\
A_{l i}^{k, h} & \equiv \sum_{v \in P C_{h}^{k}}\left(\frac{X_{t_{l}}^{v, k} X_{t_{i}}^{v, k}}{\left(N_{t_{j}}^{v, k}\right)^{2}}\right),
\end{aligned}
$$

in which, $i, l<j$. Using the above definitions, the optimal values of $\theta_{j, i}^{k, h} \mathrm{~s}$ can be written as,

$$
\Rightarrow\left(\vec{\theta}^{k, h}\right)=\left(\mathbf{A}^{k, h}\right)^{-1}\left(\vec{B}^{k, h}\right) .
$$

Eq. 20 gives the final optimal values of $\theta_{j, i}^{k, h}$ s by using the matrices defined in Eq. 19. If the matrix $A$ is not invertible; we need to reduce the dimension of $\vec{\theta}$ which means that we correlate the number of future requests to a fewer number of time intervals in the past.

\section{Enhancing MRBF model}

ML model considers the same set of parameters for all videos in each popularity class and user groups. Pinto et al. [29] proposed another model called MRBF that enhances ML by introducing a new term that is dependent on the similarities between the popularity growth of different videos. The MRBF model still introduces some global parameters for all videos. Pinto et al. utilised Gaussian Radial Basis Functions (RBFs) [13] to calculate the similarity between two videos. RBF is defined as follows,

$$
R B F_{j}^{v_{1}}\left(v_{2}\right)=\exp \left(-\frac{\left\|\vec{X}_{j}^{v_{2}, k}-\vec{X}_{j}^{v_{1}, k}\right\|^{2}}{2 \sigma^{2}}\right),
$$

in which, $v_{1}$ and $v_{2}$ have to be selected from the same popularity class; and $\sigma$ is a parameter that needs to be trained. For a specific time $t_{j}, \vec{X}^{v, k}$ is defined as,

$$
\vec{X}_{j}^{v, k}=\left(X_{t_{1}}^{v, k}, X_{t_{2}}^{v, k}, \cdots, X_{t_{j-1}}^{v, k}\right)
$$

where similar to the model based on Multivariate Linear regression, $X_{t_{i}}^{v, k}$ is the number of requests received for video $v$ in the time interval $\left[t_{i-1}, t_{i}\right]$ in group $G_{k}$. Using our proposed approach, the MRBF model can be enhanced such that its model parameters are a function of user groups and popularity classes. So, we have,

$$
\widehat{N}_{t_{j}}^{v, k}=\vec{\theta}^{k, h} \cdot \vec{X}_{j}^{v, k}+\sum_{v^{\prime} \in P C_{h}^{k}} \omega_{v^{\prime}}^{k, h} \cdot R B F_{j}^{v^{\prime}}(v) .
$$


where, $\vec{\theta}^{k, h}$ s and $\omega_{v^{\prime}}^{k, h}$ s are the model parameters. In Eq. 23, the first part is the Multivariate Linear regression model and the second part is the result of considering the similarity between the popularity distributions of different videos.

\section{Optimal values of $\vec{\theta}^{k, h}$ and $\omega_{v}^{k, h}$ parameters}

To find coefficients $\vec{\theta}^{k, h}$ and $\omega_{v}^{k, h}$, we follow the similar procedure that was presented for the previous models. To have a compact form of Eq. 22, one can define the below vectors,

$$
\begin{aligned}
\vec{X}_{j}^{v, k^{\star}} & \equiv\left(\vec{X}_{j}^{v, k}, R B F_{j}^{v}\left(v_{1}\right), R B F_{j}^{v}\left(v_{2}\right), \cdots, R B F_{j}^{v}\left(v_{n}\right)\right), \\
\vec{\theta}^{k, h^{\star}} & \equiv\left(\vec{\theta}^{k, h}, \omega_{v_{1}}^{k, h}, \omega_{v_{2}}^{k, h}, \cdots, \omega_{v_{n}}^{k, h}\right) .
\end{aligned}
$$

In the above formulas, $n$ is the number of videos in the training set. Using Eq. 24, the model in Eq. 23 may be written as follows,

$$
\widehat{N}_{t_{j}}^{v, k}=\vec{\theta}^{k, h^{\star}} \cdot \vec{X}_{j}^{v, k^{\star}} .
$$

Similar to Eq. 18, one can obtain the RSE of the predictions made by Eq. 23 in each user group $k$ and popularity class $h$. Minimising RSE with respect to the components of $\vec{\theta}^{\star}$ may result in the optimal values of the coefficients in Eq. 23. Since the number of parameters in MRBF model is larger than ML model, it is more likely that over-fitting happens for this model in a given training set. To avoid this, Pinto et al. utilised Ridge regression [13]. Ridge regression penalises those solutions with large norms. Considering this, a new term is added to RSE; and the expression that should be optimised is written as,

$$
R S E=\frac{1}{\left|v \in P C_{h}^{k}\right|} \sum_{v \in P C_{h}^{k}}\left(\frac{\widehat{N}_{t_{j}}^{v, k}}{N_{t_{j}}^{v, k}}-1\right)^{2}+\alpha\left\|\vec{\theta}^{k, h^{\star}}\right\|^{2} .
$$

We need to find the optimal values of $\alpha$ Eq. 26 and $\sigma$ Eq. 21 in the above-presented model. To do this, Pinto et al. tested a set of different values for these parameters and found the best values in that set that give the smallest RSE. In this paper, we use a similar approach. Testing a set of values in a stochastic process, we found that 0.0002 and 2 are the best values for $\alpha$ and $\sigma$, respectively.

For finding the other parameters of the model, we utilise the same method as the previous model. Taking derivative of RSE with respect to $\theta_{i}^{k, h^{\star}}$ and doing some simplifications, one may obtain the optimal values of $\theta_{i}^{k, h^{\star}} \mathrm{s}$ as follows,

$$
\left(\vec{\theta}^{k, h^{\star}}\right)=\left(\mathbf{A}^{k, h^{\star}}\right)^{-1}\left(\vec{B}^{k, h^{\star}}\right),
$$

in which,

$$
\begin{aligned}
B_{i}^{k, h^{\star}} & \equiv \frac{1}{\left|v \in P C_{h}^{k}\right|} \sum_{v \in P C_{h}^{k}} \frac{X_{t_{i}}^{v, k^{\star}}}{N_{t_{j}}^{v, k}}, \\
A_{l i}^{k, h^{\star}} & \equiv \frac{1}{\left|v \in P C_{h}^{k}\right|} \sum_{v \in P C_{h}^{k}}\left(\frac{X_{t_{l}}^{v, k^{\star}} X_{t_{i}}^{v, k^{\star}}}{\left(N_{t_{j}}^{v, k}\right)^{2}}\right)+\alpha \delta_{l i} .
\end{aligned}
$$

In Eq. 28, $\delta_{k i}$ is Kronecker delta and comes from the derivative of $\left\|\vec{\theta}^{k, h^{\star}}\right\|$ with respect to $\theta_{i}^{k, h^{\star}}$.

In this section, we first discussed the proposed grouping and video popularity classification techniques and then explained how these techniques are integrated to enhance three existing 
Improving the Accuracy of the Video Popularity Prediction Models through User Grouping and Video Popularity Classification

popularity prediction methods. Through the next section we benchmark the accuracy of the enhanced solutions with the standard popularity prediction methods based on SH, ML and MBRF.

\subsection{Computational complexity of the proposed technique}

The computations of the proposed technique are done within two parts; that are training and prediction phases. In the following, we discuss the parts and compare our proposed technique with the existing ones in this regard.

Prediction phase: This phase is done online and determines how fast an algorithm is able to predict the future popularity. A big difference between the speeds of prediction phases of two predictive algorithms causes a big difference between the speed of their reaction to the future situations. The point is that the computations needed for the prediction phase of our proposed mechanism approximately equals the computations needed for the prediction phase of the existing algorithm multiplied by the number of groups (which is around 5).

Training phase: This phase is done offline and does not affect the speed of an algorithm when it is going to react to a future situation. The main proportion of added computational complexity belongs to this part. However, we can argue that as this part is done offline the added complexity can be disregarded. To give an insight on the added time complexity of this phase, one needs to note that this phase includes two more parts, i.e. hierarchical clustering and video classification, in comparison with the existing models.

The complexity of hierarchical clustering is $O\left(n^{2} \log n\right)$ in which $n$ is number of users. Since the hierarchical clustering needs to be done $L_{\max }$ (which is given in section 4.1) times the total time complexity is $O\left(L_{\max } n^{2} \log n\right)$.

To obtain the time complexity of the video classification part, one needs to note that obtaining $N T R S E_{i}^{k}$ requires $O\left(C^{2}(C+M)\right)$ in which $\mathrm{C}$ is number of the parameters of the utilised model and $\mathrm{M}$ is number of videos in training set. Since $N T R S E_{i}^{k}$ needs to be calculated for each group and $I_{\text {max }}$ (which is given in section 4.2) times, the time complexity of this part is $O\left(\left|G_{k}\right| * I_{\max } * C^{2}(C+M)\right.$ ).

\section{DATASET AND EXPERIMENTAL RESULTS}

For the evaluation, we need a dataset of user requests made for a number of videos. A 5-fold cross-validation technique was utilised to evaluate the model. We randomly split the data for all videos into 5 parts. Each time, we used 4 folds to train the parameters of the proposed models and the remaining fold to test the models.

In each folding, using the proposed grouping method, the users who had requested similar videos in the past were assigned to the same group. Next, we used the classification technique to find the above-explained popularity growth classes. After that, the popularity prediction models can be used for predicting the future popularity of the videos in the test fold. We repeated all the described process 5 times. Finally, the final results were the average of the results in all folds.

We first describe the dataset used in this paper and then briefly discuss the results of the grouping method and video classification technique. After that, we discuss the improvements in the accuracy of the popularity prediction models. We compare our results with the results of the baseline algorithms.

\subsection{Dataset}

We used a dataset of one-month video request records of $\mathrm{BBC}$ iPlayer ${ }^{2}$ users. In the dataset, request time, video ID, anonymised user ID and duration of time that each user has spent on each specific video have been recorded. The dataset is provided by the BBC as a part of a collaboration with

\footnotetext{
${ }^{2}$ http://www.bbc.co.uk/iplayer
} 
the 5G Innovation Center (5GIC) at the University of Surrey. The original dataset comprises more than 100 million distinct entries; to reduce the size and at the same time have a comprehensive subset, 56083 users were randomly selected amongst all users. After that, all the information about requests made by this subset of users was extracted from the dataset.

The subset consists of $1,861,769$ requests that have been made for 20,946 unique videos. Since one needs to have sufficient history of user requests to build the user groups, we removed those users who had made less than 10 requests within one month. Also, we selected those videos that had been requested more than 40 times during this period. The subset after the elimination contains 26,706 unique users and 3018 unique videos. The subset includes 793468 requests made for the remaining 3018 videos. The average number of requests received for videos is $\sim 250$ and the most popular video is requested more than 4500 times.

It is worth to be noted that we have randomly selected the above-mentioned number of users from a much larger dataset which is more than $\sim 80$ times larger than the used dataset in our paper. As the process of user selection has been random, it can be concluded that if a video is requested $\sim 250$ times in the sub dataset, it is requested in the main dataset $\sim 20000$ times. In addition, the random selection of users and the large number of selected users indicate that exiting patterns in the main dataset and in the sub dataset are similar. As the result, it is not expected that the presented improvements in this paper change a lot for the main dataset.

\subsection{Evaluation results}

Parameters of the conventional SH, ML and MBRF models are trained over all users in the training set. Whereas the parameters of their enhancements are to be calculated for each specific user group and popularity class. The trained parameters are employed to forecast the number of requests in the test set.

We add all the number of requests that are predicted by each enhanced popularity prediction method in each user group till the time $t_{j}$ for a specific video $v$ (i.e. $\widehat{N}_{t_{j}}^{v, k}$ ) to find the total number of requests received for $v$. In the evaluation, the calculated total number of requests is compared with the predicted number of requests by the conventional SH, ML and MRBF methods. To have a fair comparison, each model from the prior state-of-the-art is compared against one of our newly developed models that is the enhancement of the same conventional model.

It should be noted that the time of the first request can vary in different groups and this difference should be considered when calculating the total number of received requests.

Finally, we compare the RSEs, defined in Eq. 14, of the predictions made to evaluate the impact of the proposed approach on the performance of the V-PPMs.

\section{User groups}

According to the proposed technique in Section 4.1, a hierarchical tree was constructed for our set of users.

As explained in section 4.1, we need to set a lower bound for the number of users in each group. We considered 100 as the minimum acceptable number of users in each group to penalise the creation of the small groups in the clustering process.

We used MATLAB to construct hierarchical tree for our set of users. To link the users (objects), we utilised "complete" ${ }^{3}$ approach.

After that, the total entropy for different cutting levels in $\left[0, L_{\max }\right]$ was calculated. In different foldings, the proposed grouping technique found different number of clusters. The obtained number

\footnotetext{
${ }^{3}$ In the most basic level, the complete approach pairs an object to its nearest object. Then, the approach considers the furthest distance between objects of two previously paired objects as the distance between that two pairs.
} 
Improving the Accuracy of the Video Popularity Prediction Models through User Grouping and

Video Popularity Classification

of groups in different foldings ranges from 4 to 7 . This is down to the point that the best number of clusters may vary for different training datasets.

\section{Video popularity classes}

This section discusses the results of our proposed video popularity classification technique. The video popularity classification technique determines the popularity levels that separate different popularity classes in each user group based on their received early popularities.

We set the minimum number of videos in each popularity class to 15 and the maximum number of popularity classes for each user group to 2 . The maximum number of popularity classes was determined by looking at the number of received requests in each user group. In all user groups, there is at most one clear gap that separates the videos, corresponding to high and low popular videos.

Considering the above assumptions, the classification technique resulted in different number of popularity classes ( 1 or 2 classes) in different groups.

\subsection{Discussion}

\section{Evaluation settings}

We need to pre-set the length of time intervals (i.e. $\left[t_{i-1}, t_{i}\right]$.) for the mentioned models. For user generated videos, the typical time interval that is used for the evaluations is about 1 day [29] [35]. However, since the used dataset in this paper contains on-demand videos and the popularity of the on-demand videos grows much faster than the UGC videos, we set this length to 30 minutes. The maximum time in which we predict the number of received requests is set to 3 days after the released time. In the next step, we normalised all the points in the time axis by the maximum time; so, points 0 and 1 in the time axis show time of the release time and 3 days after release time of the videos.

\section{The evaluation of our proposed model}

We use RSE to compare the accuracy of the predictions made by the baseline model and our proposed solution.

Fig. 2 presents the box plot of the RSEs for all the predictions. It is evident that the enhanced models by our approach result in a considerable reduction in the range of RSEs in comparison with the conventional models. The highest improvement is related to the SH model. The median, in the graph related to SH model, has been reduced from 0.0144 to 0.0056 (relatively, $61 \%$ improvement). For ML model, the median has been improved from 0.0086 to 0.0046 which is equivalent to $\sim 45 \%$ improvement. For MRBF model, we observe the lowest reduction $(\sim 24 \%)$ in the median (from 0.0411 to 0.0046$)$. Table 1 summarises the improvements discussed above.

Table 1. Relative improvements in terms of median

\begin{tabular}{|c|c|c|c|}
\hline & SH model & ML model & MRBF model \\
\hline Baseline model & 0.0144 & 0.0086 & 0.0083 \\
\hline Enhanced model & 0.0056 & 0.0046 & 0.0063 \\
\hline Relative improvement & $61 \%$ & $45 \%$ & $24 \%$ \\
\hline
\end{tabular}

The box plots presented in Fig. 2 shows that in total the enhanced models by our proposed approach outperforms the conventional SH, ML and MRBF models.

It is worth to be noted that the proposed classification technique actually divides videos into two classes of popular and unpopular videos. If dividing videos into popular and unpopular classes 


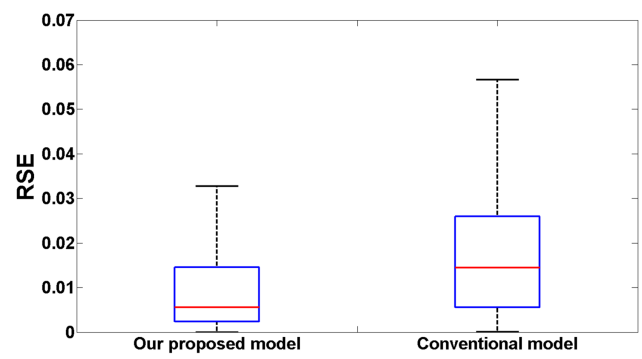

(a) Ratio of RSEs of our SH-based model and $\mathrm{SH}$ model

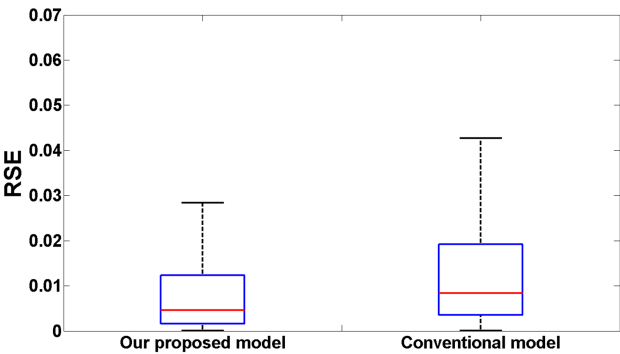

(b) Ratio of RSEs of our ML-based model and ML model

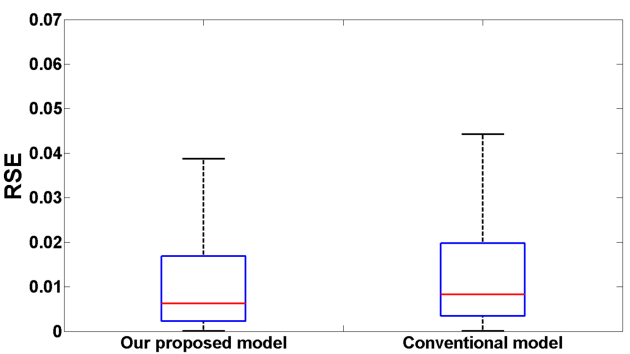

(c) Ratio of RSEs of our MRBF-based model and MRBF model

Fig. 2. Box plot of RSEs of the predictions. Each box represents the RSE of the predictions made by the models (first box is related to the enhanced model and second one is related to the conventional model). Comparing the range and median of box plots can be utilised to evaluate the impact of our proposed approach on the performance of the conventional models. In terms of median, the proposed approach has relatively improved the median of SH model by $61 \%$, the median of ML model by $45 \%$ and the median of MRBF model by $24 \%$.

results in improvements in the model accuracy, the technique does this classification; otherwise, it does not. As a fact, only about $10 \%$ of videos become popular. As the result, the number of videos in the unpopular class is much more than the number of videos in the popular class. So, the values of model parameters is much closer to their values for unpopular videos than their values for popular videos. Dividing videos into popular and unpopular classes causes that V-PPM becomes able to more accurately find the values of model parameters for popular videos. The direct result of this discussion is that employing the proposed classification technique enables a model to better estimate the future popularity of popular videos.

Considering the above-explained points, we have conducted a new experiment to find the impact of each factor on predicting popularity of popular videos and validated the results by 5 -fold cross validation. The results show that for popular videos classifying videos based on their popularities 


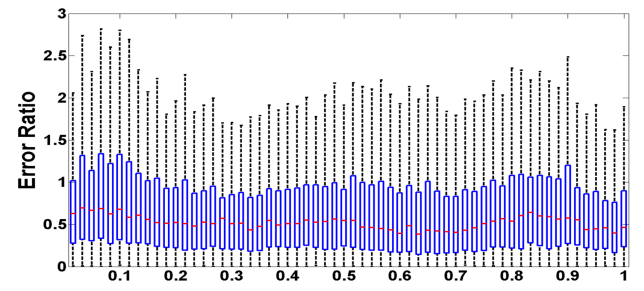

(a) Ratio of RSEs of our $\mathrm{SH}$-based model and $\mathrm{SH}$ model

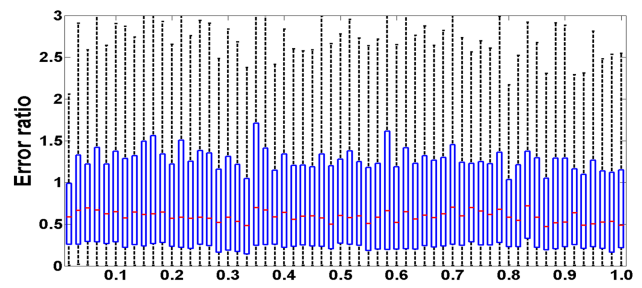

(b) Ratio of RSEs of our ML-based model and ML model

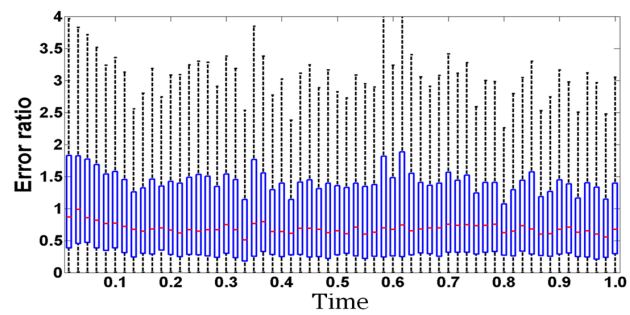

(c) Ratio of RSEs of our MRBF-based model and MRBF model

Fig. 3. Box plot of RSE ratios of all the predictions. Each ratio is calculated as the RSE of each prediction made by our proposed approach divided by the RSE of the prediction made by conventional model. Then, we have depicted the box plot of all ratios in each specific time. As it is shown, the majority of the ratios are smaller than 1 and show that our proposed approach most of the times work better than the conventional model. The SH model has shown the best improvements in the Ratio of the RSEs which is in agreement with the results presented in the figure 2 .

results in $9 \%$ and $15 \%$ improvements in terms of median in the performance of SH and ML models enhanced by our grouping technique.

It needs to be stressed that user grouping has greater impact on the observed improvement in the accuracy of popularity prediction and improves the accuracy of predictions made for all videos.

In Fig. 3, each panel shows the ratios of RSEs of the conventional techniques and their enhancements by our approaches. Error ratios less than 1 imply that the RSEs have decreased by applying the proposed approach. Through majority of the boxes ( $70 \%$ of them), it is evident that our proposed approach improves the performance of the conventional models. The medians of the ratio of the errors for all the three models in different time intervals are less than 1 . For the $\mathrm{SH}$ and ML-based models, these ratios are more stable than the MRBF model and have the smallest ranges. The biggest ranges are related to MRBF model. Hence, the proposed technique improves the SH and ML models more than MRBF. It can be due to the point that the MRBF model has much larger number of parameters with respect to the other models.

To give an idea about the overall improvements of the predictive models, we propose to calculate the average of the relative improvement as follows. For this purpose, we compute the mean of all 
Table 2. Mean relative improvements

\begin{tabular}{|l|c|c|c|}
\hline & SH model & ML model & MRBF model \\
\hline $\begin{array}{l}\text { Overall (relative } \\
\text { improvement) }\end{array}$ & $45 \%$ & $33 \%$ & $24 \%$ \\
\hline
\end{tabular}

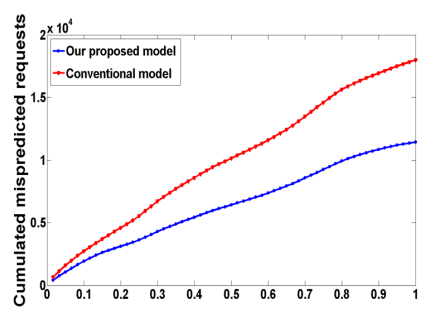

(a) SH-based model

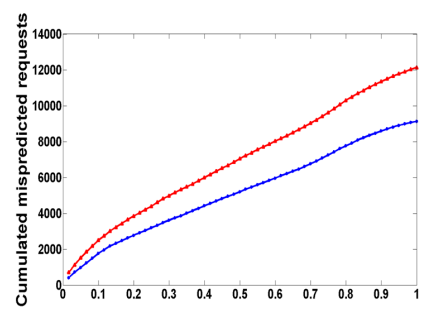

(b) ML-based model

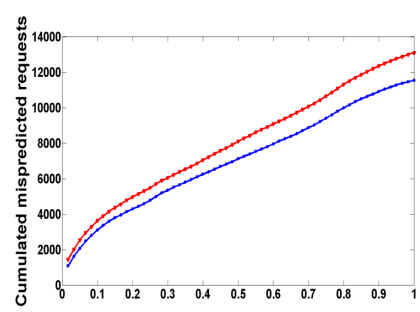

(c) MRBF-based model

Fig. 4. Accumulated miss-predicted requests calculated as the sum of requests that did not predicted by the popularity prediction models.

error ratios presented in Fig. 3. After that, we find the relative improvement by subtracting the mean from 1 . So, we have

$$
O R I=1-M E R .
$$

in Eq. 29, ORI and MER are Overall Relative Improvement and Mean of Error Ratios, respectively. Table 2 presents the overall relative improvements of all the three models. As expected, we observe the highest improvement in the accuracy of SH-based model and the lowest one in the accuracy of MRBF-based model.

Up to this point, the evaluation has not provided any information about the improvements in the exact number of the mis-predicted requests until a certain point in time (i.e. exact error). In the next section, we evaluate the above-discussed models based on the exact number of the prediction errors.

\section{Improvements in terms of cumulative error}

The evaluation provided in this section is based on cumulative errors. Cumulative error is defined as the number of requests that have been missed by a popularity prediction model. To compute the cumulative error, in each, we accumulate the number of miss-predicted requests up to a specific time and then take the average of the cumulative errors across all 5 folds of cross validation. The graphs presented in the Fig. 4 shows the explained accumulated mis-predicted requests for the proposed models based on our proposed approach against the conventional models over time. As can be seen, the curves associated with the proposed models is always below the curves related to the conventional models. This again shows that by using the proposed technique, we have made more accurate predictions for the popularity of the newly-released videos.

Based on the evaluation presented above, we can conclude that the proposed solution considerably outperforms the conventional V-PPMs. Since we have randomly selected the utilized (sub) dataset in our paper from a significantly larger (main) dataset (which is 80 times larger), observing 400 requests received for a particular video in the sub dataset means that this video is probably requested 32000 times in the main dataset. On the other hand, it is not expected that the observed accuracies in our study noticeably change for the main dataset. This is down to the point that the random 
Improving the Accuracy of the Video Popularity Prediction Models through User Grouping and Video Popularity Classification

selection of users doesn't affect the existing patterns in the popularity growth of videos that exist in the main dataset; therefore, the accuracy of a model that works based on the mentioned patterns doesn't experience a noticeable change. Considering MRBF as an example, the results presented in table 1 and 32000 requests received for the particular video in the main dataset, MRBF model and our proposed model result in $\sim 2912$ and $\sim 2528$ miss-predicted requests, respectively. The difference between these two errors increases for the popular videos with more than 3000 received requests in the sub dataset. These videos are requested $\sim 240000$ times and more in the main dataset and the number of miss-predicted requests is more than $\sim 21840$ and $\sim 18960$, respectively.

In the case of our study, the reported accuracies depends on the selected value as the time interval (explained in the section 8.3). The time interval determines how much time later we wish to predict the popularity of videos. In our paper and due to the speed of the popularity growth of on-demand videos, we have selected half an hour as the time interval. It is clear that if we increase the length of the interval, the reported accuracies diminish. In contrast, the comparison results between two models are somewhat stable. According to this, observing that our proposed solution performs better than the baseline models can lead us to conclude that our solution works better than the baselines in other situations. In brief, the reported RSE for the models in our paper would increase if we wanted to predict in the larger intervals (e.g. 3-hour intervals); as the result, the number of the miss-predicted requests would increase. However, the improvements resulted from our proposed solution remain quite stable for different situations.

\section{Limitations of the Proposed Model}

Our proposed model has a number of limitations that are discussed in this section.

First, the evaluation results presented in this paper show that $\mathrm{SH}$ model benefits the most from the proposed technique. The potential reasons are discussed in what follows. Note that the less number of model parameters of SH model in comparison with the ML and MRBF models indicates that $\mathrm{SH}$ model is more simple than ML and MRBF models. Therefore, the SH model is not as powerful as ML and MRBF models to capture the underlying patterns existing in the popularity growth of the videos.

On the other hand, the actual amount of the existing patterns has an upper bound; so, more powerful models can capture a bigger proportion of the patterns. As the result, any enhancement on a powerful model may not result in a relatively big improvement in the proportion of the patterns that can be captured by the model. Due to this, improving a powerful model is more difficult than a simple model.

Considering the simplicity of SH model and the above-discussed upper bound for the patterns, an enhancement may result in relatively bigger improvements in the performance of SH model than the ML and MRBF model. Similar discussion can be applicable for comparing the ML and MRBF models in this regard. To explain this, as MRBF model is more complex than the SH and ML models, its performance improves less than the other two models.

As the second limitation, our proposed model requires more computations than the conventional models. As discussed previously, the prediction phase is not affected a lot by this added computations; therefore, the speed of the prediction phase does not reduce noticeably. Nevertheless, we need to search for a method to reduce the amount of needed computations specially for large video streaming platforms that has a large number of users.

As the first solution to treat the discussed problem, it is worth to be noted that our proposed technique can be run distributed. We actually do not need to find user groups and video popularity classes for all users using a video streaming platform and all videos that exist on the platform. To illustrate it, the user groups and video popularity classes can be found for each geographical region (a city or even a part of city). 
As the other solution to treat the discussed problem, we need to search for more efficient methods to assign new users to the previously found user groups. One possible method is to represent the groups by their most popular videos and assign the users who have requested the popular videos of a specific group to the group. Other methods should be found and their performance and efficiency need to be tested.

The final limitation is that as dividing videos into a number of classes reduces the number of videos utilised for training model parameters, it may increase the probability of occurring over-fitting for the employed models. More complex models, e.g. ML and MRBF models, are more susceptible to this problem. utilising regularisation techniques may be a good solution for this problem.

\section{IMPLICATIONS TO IMPROVE POTENTIAL APPLICATIONS}

Our results confirms that considering user groups and video popularity classes has a significant impact on improving the accuracy of the popularity prediction models.

Based on our findings, we make a few recommendations on the deployment of V-PPMs in its most popular applications in the network and service management domain. These applications include caching systems, advertisement mechanisms and broadcasting technologies.

- Implications for Wireless networks application: The implications of our results for network operators can be listed as,

(1) Our results imply that network and service management applications should always consider to keep a limited information about previous video request of users. Such information would then be sufficient to identify both popularity classes and user groups, which significantly improve the accuracy of the predictions.

(2) Our results show that different user groups follow distinct popularity patterns and reaction times. Therefore, caching mechanisms at any moment should consider both the reaction times of different groups and their population to estimate the dynamic of the popularity of each video before predicting the popular videos for cashing.

(3) Our results reveal that videos only get popular in a subset (either a group or a few groups) of users instead of whole population. Using the knowledge about the geographical distribution of the user groups, one can more accurately estimate the spatial distribution of demand received for the popular videos. The estimated distribution of the demand is a valuable input to improve Device to Device caching systems. More specifically, decisions on caching or distributing contents should be taken based on the group-level geographical distribution of future requests that will be received for popular videos.

(4) To improve the efficiency of the broadcasting mechanisms, decisions on either broadcast, multicast or unicast a video should be taken based on the popularity of the video in each user group, the population and the geographical distribution of users in the group.

(5) As a result of observing at-least two video popularity classes, the employed V-PPM in network ought to consider at-least two video popularity classes to improve its predictions.

- Implications for marketing industries: Online advertisement is considered as a valuable source of income for content providers. The group-level popularity predictions of our approach and the characteristics of user groups can be utilised to find Better advertisement strategies.

By analyzing the content and characteristics of the popular videos in each user group, one can identify the collective characteristics, e.g. age or gender, of the users in each group. By considering these collective characteristics of the users in different groups, an advertisement can be targeted to the group of users who are potentially interested in that advertisement. 
Moreover, the knowledge about the characteristics of the user groups and content of the newly-released videos can be also utilised to recommend the most appropriate video for each advertisement. So, by this way, an advertisement may receive more number of views that is an important factor for success of an advertisement.

\section{CONCLUSION}

We proposed a novel approach for enhancing the video popularity prediction models. The proposed approach takes the underlying similarities in the requested videos by users and in the received popularity growth trend for videos into consideration.

We used the proposed approach to enhance three well-known popularity prediction models, namely SH, ML and MRBF models. The enhanced models initially build user groups with similar interests. The user grouping process is conducted through an unsupervised grouping technique. The technique is based on hierarchical clustering and chooses its optimal cut-off level by calculating the total entropy of video requests made by users in the resulted groups for a set of videos.

Within each user group, our proposed technique finds an effective criteria to classify the videos into popularity growth classes.

Considering user groups and video popularity classes, the proposed popularity prediction models find the dependencies between past and future popularity of the videos. Therefore, the optimum values of the parameters of the models are a function of user group and popularity class numbers.

In the evaluation part, we used a dataset containing user video requests gathered from the users of $\mathrm{BBC}$ iPlayer. The evaluation was accomplished by 5 -fold cross validation. The proposed grouping technique was utilised to find user groups with similar taste in video. Next, the associated popularity classes to each user group were found. To evaluate our proposed models, we compared the models with SH, ML and MRBF models. We compared the accuracy of the enhanced models with its conventional counterparts. On average for the set of all videos, we observed that the proposed approach is able to improve the SH, ML and MRBF models by $45 \%, 33 \%$ and $24 \%$, respectively. We also showed that how our proposed solution improves the conventional methods in terms of accumulated mis-predicted requests.

In spite of observing significant improvements by using the proposed grouping technique in this paper, we also need to test the impact of other clustering methods on the performance of the popularity prediction models and compare the improvements resulted from using these methods with the improvements resulted from our proposed grouping method. Some of the main clustering methods that need to be tested are k-mean, k-way graph partitioning, affinity propagation and density-based clustering techniques.

Moreover, the observed improvements needs to be checked in other use-cases and tested against other datasets. Observing the improvements for other datasets can strongly support our findings in this paper. Considering this, another future step for this research is to test our proposed techniques by other datasets.

In addition, the presented improvement results might change for popular and unpopular videos. As discussed previously, the grouping and classification factors may also have different impact for the popular and unpopular videos. Studying the improvements and impact of each factor for popular and unpopular videos is the other future step of this study.

It is worth to be mentioned that our proposed approach adds more computational complexity in training phase but results in a better accuracy. The added complexity is performed offline and does not affect the performance of the prediction phase of the proposed models. 


\section{ACKNOWLEDGMENTS}

This research is supported by the 5G Innovation Centre at the University of Surrey. The authors would like to thank the $\mathrm{BBC}$ team for providing the on-demand access data. We also would like to thank Dr Chang Ge for providing pre-analysis on The BBC dataset to remove the missing data They also would like to thank Gerry Foster for stimulating discussions and Dr Vahid Heydari Fami Tafreshi for his comments and suggestions.

\section{REFERENCES}

[1] Zlatka Avramova, Sabine Wittevrongel, Herwig Bruneel, and Danny De Vleeschauwer. 2009. Analysis and modeling of video popularity evolution in various online video content systems: Power-law versus exponential decay. In Evolving Internet, 2009. INTERNET'09. First International Conference on. IEEE, 95-100.

[2] Jesús Bobadilla, Fernando Ortega, Antonio Hernando, and Abraham Gutiérrez. 2013. Recommender systems survey. Knowledge-based systems 46 (2013), 109-132.

[3] Youmna Borghol, Siddharth Mitra, Sebastien Ardon, Niklas Carlsson, Derek Eager, and Anirban Mahanti. 2011. Characterizing and modelling popularity of user-generated videos. Performance Evaluation 68, 11 (2011), 1037-1055.

[4] Meeyoung Cha, Haewoon Kwak, Pablo Rodriguez, Yong-Yeol Ahn, and Sue Moon. 2009. Analyzing the video popularity characteristics of large-scale user generated content systems. IEEE/ACM Transactions on Networking (TON) 17, 5 (2009), 1357-1370.

[5] Xu Cheng, Cameron Dale, and Jiangchuan Liu. 2007. Understanding the characteristics of internet short video sharing: YouTube as a case study. arXiv preprint arXiv:0707.3670 (2007).

[6] Xu Cheng, Cameron Dale, and Jiangchuan Liu. 2008. Statistics and social network of youtube videos. In Quality of Service, 2008. IWQoS 2008. 16th International Workshop on. IEEE, 229-238.

[7] Delia Ciullo, Valentina Martina, Michele Garetto, and Emilio Leonardi. 2015. How much can large-scale video-ondemand benefit from users' cooperation? IEEE/ACM Transactions on Networking (TON) 23, 6 (2015), 1846-1861.

[8] Riley Crane and Didier Sornette. 2008. Robust dynamic classes revealed by measuring the response function of a social system. Proceedings of the National Academy of Sciences 105, 41 (2008), 15649-15653.

[9] Riley Crane, Didier Sornette, et al. 2008. Viral, Quality, and Junk Videos on YouTube: Separating Content from Noise in an Information-Rich Environment.. In AAAI Spring Symposium: Social Information Processing. 18-20.

[10] Jeroen Famaey, Tim Wauters, and Filip De Turck. 2011. On the merits of popularity prediction in multimedia content caching. In Integrated Network Management (IM), 2011 IFIP/IEEE International Symposium on. IEEE, 17-24.

[11] Flavio Figueiredo. 2013. On the prediction of popularity of trends and hits for user generated videos. In Proceedings of the sixth ACM international conference on Web search and data mining. ACM, 741-746.

[12] Giulia Fontanini, Marco Bertini, and Alberto Del Bimbo. 2016. Web video popularity prediction using sentiment and content visual features. In Proceedings of the 2016 ACM on International Conference on Multimedia Retrieval. ACM, 289-292.

[13] Jerome Friedman, Trevor Hastie, and Robert Tibshirani. 2001. The elements of statistical learning. Vol. 1. Springer series in statistics Springer, Berlin.

[14] Thomas Hayes. 2006. Scalable system and method for predicting hit music preferences for an individual. US Patent App. 11/253,421.

[15] Minh X Hoang, Xuan-Hong Dang, Xiang Wu, Zhenyu Yan, and Ambuj K Singh. 2017. GPOP: Scalable Group-level Popularity Prediction for Online Content in Social Networks. In Proceedings of the 26th International Conference on World Wide Web. International World Wide Web Conferences Steering Committee, 725-733.

[16] Wei-jen Hsu, Debojyoti Dutta, and Ahmed Helmy. 2012. Structural analysis of user association patterns in university campus wireless lans. IEEE Transactions on Mobile Computing 11, 11 (2012), 1734-1748.

[17] Anil K Jain. 2010. Data clustering: 50 years beyond K-means. Pattern recognition letters 31, 8 (2010), $651-666$.

[18] Liping Jing, Michael K Ng, and Joshua Zhexue Huang. 2007. An entropy weighting k-means algorithm for subspace clustering of high-dimensional sparse data. IEEE Transactions on knowledge and data engineering 19, 8 (2007).

[19] Chenyu Li, Jun Liu, and Shuxin Ouyang. 2016. Characterizing and predicting the popularity of online videos. IEEE Access 4 (2016), 1630-1641.

[20] Liying Li, Guodong Zhao, and Rick S Blum. 2018. A Survey of Caching Techniques in Cellular Networks: Research Issues and Challenges in Content Placement and Delivery Strategies. IEEE Communications Surveys \& Tutorials (2018).

[21] Mark Junjie Li, Michael K Ng, Yiu-ming Cheung, and Joshua Zhexue Huang. 2008. Agglomerative fuzzy k-means clustering algorithm with selection of number of clusters. IEEE transactions on knowledge and data engineering 20, 11 (2008), 1519-1534. 
[22] Jiye Liang, Xingwang Zhao, Deyu Li, Fuyuan Cao, and Chuangyin Dang. 2012. Determining the number of clusters using information entropy for mixed data. Pattern Recognition 45, 6 (2012), 2251-2265.

[23] Hai-yong Liao and Michael K Ng. 2009. Categorical data clustering with automatic selection of cluster number. Fuzzy Information and Engineering 1, 1 (2009), 5-25.

[24] Changsha Ma, Zhisheng Yan, and Chang Wen Chen. 2017. LARM: A lifetime aware regression model for predicting youtube video popularity. In Proceedings of the 2017 ACM on Conference on Information and Knowledge Management ACM, 467-476.

[25] Kianoosh Mokhtarian and Hans-Arno Jacobsen. 2017. Flexible caching algorithms for video content distribution networks. IEEE/ACM Transactions on Networking (TON) 25, 2 (2017), 1062-1075.

[26] Joseph Kee-Yin Ng, Victor Chung-Sing Lee, and Chui Ying Hui. 2008. Client-side caching strategies and on-demand broadcast algorithms for real-time information dispatch systems. IEEE Transactions on Broadcasting 54, 1 (2008), 24-35.

[27] Amandianeze O Nwana, Salman Avestimehr, and Tsuhan Chen. 2013. A latent social approach to youtube popularity prediction. In Global Communications Conference (GLOBECOM), 2013 IEEE. IEEE, 3138-3144.

[28] Shuxin Ouyang, Chenyu Li, and Xueming Li. 2016. A Peek Into the Future: Predicting the Popularity of Online Videos. IEEE Access 4 (2016), 3026-3033.

[29] Henrique Pinto, Jussara M Almeida, and Marcos A Gonçalves. 2013. Using early view patterns to predict the popularity of youtube videos. In Proceedings of the sixth ACM international conference on Web search and data mining. ACM, 365-374.

[30] Suman Deb Roy, Tao Mei, Wenjun Zeng, and Shipeng Li. 2013. Towards cross-domain learning for social video popularity prediction. IEEE Transactions on multimedia 15, 6 (2013), 1255-1267.

[31] Bo Shu, Wei Chen, Zhendong Niu, Changmin Zhang, and Xiaotian Jiang. 2013. A Novel Method for Identifying Optimal Number of Clusters with Marginal Differential Entropy. In International Conference on Web-Age Information Management. Springer, 371-382.

[32] Gabor Szabo and Bernardo A Huberman. 2010. Predicting the popularity of online content. Commun. ACM 53, 8 (2010), 80-88.

[33] Zhiyi Tan, Yanfeng Wang, Ya Zhang, and Jun Zhou. 2016. A novel time series approach for predicting the long-term popularity of online videos. IEEE Transactions on Broadcasting 62, 2 (2016), 436-445.

[34] Alexandru Tatar, Marcelo Dias de Amorim, Serge Fdida, and Panayotis Antoniadis. 2014. A survey on predicting the popularity of web content. Fournal of Internet Services and Applications 5, 1 (2014), 8.

[35] Jiqiang Wu, Yipeng Zhou, Dah Ming Chiu, and Zirong Zhu. 2016. Modeling dynamics of online video popularity. IEEE Transactions on Multimedia 18, 9 (2016), 1882-1895.

[36] Tingyao Wu, Michael Timmers, Danny De Vleeschauwer, and Werner Van Leekwijck. 2010. On the use of reservoir computing in popularity prediction. In Evolving Internet (INTERNET), 2010 Second International Conference on. IEEE, 19-24.

[37] Lexing Xie, Apostol Natsev, Xuming He, John R Kender, Matthew Hill, and John R Smith. 2013. Tracking large-scale video remix in real-world events. IEEE Transactions on Multimedia 15, 6 (2013), 1244-1254.

[38] Jie Xu, Mihaela van der Schaar, Jiangchuan Liu, and Haitao Li. 2015. Forecasting popularity of videos using social media. IEEE Journal of Selected Topics in Signal Processing 9, 2 (2015), 330-343.

[39] Chengang Zhu, Guang Cheng, and Kun Wang. 2017. Big Data Analytics for Program Popularity Prediction in Broadcast TV Industries. IEEE Access 5 (2017), 24593-24601.

Received November 2018 